\title{
Location estimation of radio transmitters based on spatial interpolation of RSS values
}

\author{
Valentin Rakovic, Marko Angjelicinoski, Vladimir Atanasovski and Liljana Gavrilovska \\ Faculty of Electrical Engineering and Information Technologies \\ Ss Cyril and Methodius University in Skopje \\ Skopje, Macedonia \\ liljana@feit.ukim.edu.mk
}

\begin{abstract}
Detection of potential transmitters' location is one of the vital aspects for efficient practical deployment of secondary spectrum access solutions. It requires accurate and up-to-date radio environmental estimation. Spatial interpolation techniques can allow partial or complete insight into the radio field, the interference and the possible geo-locations of various field transmitters depending on the number of radio measurements performed in sparse locations. This paper presents an effective solution based on spatially interpolated Received Signal Strength (RSS) values for location estimation of radio transmitters, which operates on Radio Interference Field (RIF) maps obtained by interpolating measurement data from $N$ sparsely distributed sensors. In contrast to the known range based localization methods the developed technique also achieves higher computational efficiency. The performance analysis shows that the proposed method is suitable for both outdoor and indoor environments and is capable of reliable detection of multiple sources even for low number of sensors.
\end{abstract}

Keywords- Radio intereference field, Localization, Cognitive radio, Interpolation.

\section{INTRODUCTION}

The emerging secondary spectrum usage notion based on the Cognitive Radio (CR) paradigm provides viable solution to the spectrum scarcity problem. The key challenge within is the accurate detection and identification of vacant spectrum opportunities. The Radio Environmental Map (REM) concept fosters the process of spectrum holes identification beyond the existing detection-based methods [1]. It provides the secondary CR systems with partial or complete insight into the radio interference distribution over an inspected area of interest, location of potential transmitters and other relevant information necessary for reliable operation of CR networks (CRNs).

With the latest advances in the area of CRN deployments (e.g. cognitive femto-cells), the transmitter localization process has become a crucial method that enables the process of dynamic spectrum access, self-organization and cooperation. The localization process carries valuable information for both Primary User (PU) protection and Secondary User (SU) service provision. PU localization enables the SU systems to achieve higher spectrum utilization while maintaining the required PU protection, whereas the SU localization provides better cooperation between multiple SU transmitters and increases the efficiency of the spectrum sharing concept.

This paper presents a simple and effective multiple radio transmitters localization method based on the spatially interpolated Received Signal Strength (RSS) values. The method operates on the Radio Interference Field (RIF) maps obtained by interpolating data measurement from a number of sparsely distributed sensors in the area of interest. It tracks the temporal changes of the monitored radio environment in order to detect the activation of new interfering transmitters. The method adapts to the temporal changes in the radio environment by searching for a solution which optimizes some predefined cost function. In contrast to the known localization methods, the proposed method is computationally efficient and does not require complex hardware solutions like antenna arrays (AoA based techniques) or high fidelity synchronization (TDoA based techniques). Additionally, the method is capable of detecting multiple transmitters without increasing its computational complexity or decreasing its precision.

The rest of the paper is organized as follows. Section II gives an overview of the related work on multiple transmitter localization techniques. Section III defines the system model, while section IV elaborates on the proposed localization method. Section V provides performance analysis of the proposed method. Finally, section VI concludes the paper.

\section{RELATED WORK}

The problem of multiple transmitters localization attracts increased interest lately [2]. The most common classification of known multiple radio transmitters localization techniques is into the classes of range-free and range-based approaches.

Range-free approaches use topological information to infer the locations of the multiple targets, therefore saving any special hardware costs, and trading off the accuracy and scalability of the location estimates [1,3]. Range-based localization approaches derive the position of the unknown transmitters using range estimates from location known anchors, such as RSS, angle of arrival (AOA), time of arrival (TOA) or time difference of arrival (TDOA). In general, accurate range measurements require special hardware. 
Therefore, less accurate but easily available RSS-based measurements are used extensively in many localization algorithms under probabilistic models. Ref. [2] discusses some of the most popular location estimators. Maximum Likelihood (ML) is a common localization approach, that provides transmitter location estimates with limited accuracy because it results in a non-convex optimization problem. Ref. [4] circumvents the non-convexity of the conventional ML solution by applying the semi-definite relaxation to the ML estimator and develops a convex estimator. Similarly, [5] elaborates a novel approximate ML approach (referred to as Weighted Least Squares - WLS) that alleviates the nonconvexity of the ML technique by reformulating the problem of localization under the equivalent exponential transformation of the conventional path loss measurement model and the unscented transformation. Ref. [6] presents a localization solution specifically designed for dense indoor environments with unknown path loss exponent. This algorithm leverages between the computational cost and localization accuracy by establishing a neighbor selection scheme based on the Voronoi diagram to identify a subset of sensors to participate in localization. Linear Least Squares (LLS) localization approach as Best linear Unbiased Estimator (BLUE) is presented in [7]. Additionally, ref. [8] derives Maximum Likelihood (ML) localization algorithm and the associated performance bounds for jointly estimating a transmitter's position, orientation, beam width and transmit power, as well as the environment's path loss exponent using RSS measurements.

Much of the work that has been done to address the localization problem points out the limited accuracy of the majority of localization algorithms (when dealing with reasonable processing power) and the ever-increasing requirements for computational resources by the underlying optimization algorithms (when trying to reduce the localization error). However, there is an increasing number of secondary spectrum usage scenarios that significantly relax the localization precision constraints and require only target detection and, if possible, coarse location estimation in a given area. This paper targets such scenarios by developing simple and efficient localization technique that requires modest computational power while providing effective target presence and location information.

The following section will explain in more details the envisioned target scenario for the subsequent location estimation method based on spatial interpolation.

\section{TARGET SCENARIO}

The proposed method for presence detection and location estimation targets a similar scenario as the one depicted on Fig. 1 and 2.

Fig. 1a represents an area of interest that has $M$ active interferers at a specific time moment. In the case of Fig. 1, there are two transmitters denoted as Transmitter 1 and Transmitter 2. Fig. 1b shows the same system after a certain time period when an additional transmitter denoted as Transmitter 3 is activated. Fig. $2 \mathrm{a}$ and $2 \mathrm{~b}$ show the RIF maps over the area of interest, before and after the appearance of
Transmitter 3, respectively. The RIFs are obtained by interpolating the measurement data from $N$ spatially distributed sensors using the modified Shepherd's method [9] for spatial interpolation. The new interferer causes changes in the RIF (as evident from Fig. 2), i.e. the distribution of the interference power over the area of interest changes due to the activation of Transmitter 3 .

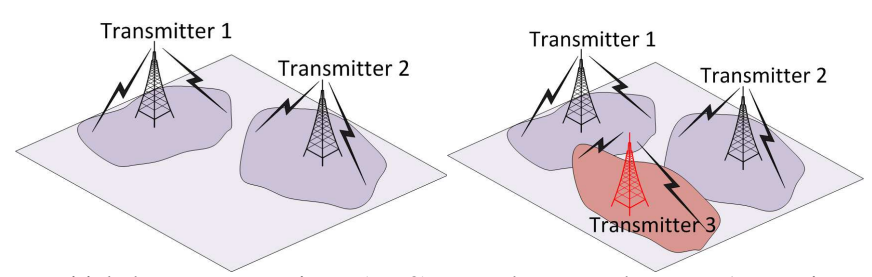

a.Initial phase, $\mathrm{M}$ transmitters $(\mathrm{M}=2)$

b. Latter phase, $M+1$ ransmitters Figure 1. Target scenario

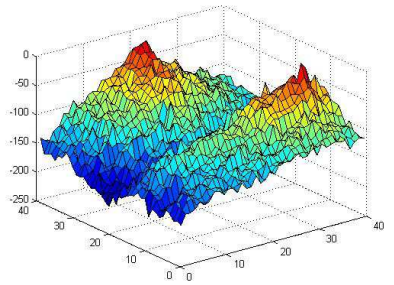

a.Initial phase, $\mathrm{M}$ transmitters $(\mathrm{M}=2)$

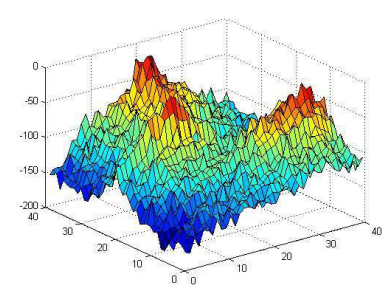

b. Latter phase, $\mathrm{M}+1$ ransmitters Figure 2. RIF for the targeted scenario on Fig. 1
The RIF changes can be efficiently tracked by defining an appropriate qualitative measure which will be referred to as a tracking metric. This tracking metric gives information on the interferers presence in the area of interest. Moreover, it is possible to refine the tracking metric in order to locate regions in the area of interest where the highest amount of the RIF changes are cumulated, thus providing estimates of the interferers location. The analysis in the following section assumes an approach that conducts a statistical analysis of the changes of the radio interference level in different points when adding new interferers in the area of interest. It relies on the idea that the new interferers cause higher increase of the interference level at nearby points than at distant points.

\section{INTERFERENCE LEVEL BASED LOCATION ESTIMATION OF MULTIPLE TRANSMITTERS}

This section gives a thorough theoretical analysis of the problem of spatial interpolation based location estimation of a potential interferer. It explains the used assumptions and gives an analytical modeling of the tackled problem.

\section{A. RIF based localization with fixed regions}

The inspected area of interest (i.e. the target scenario previously analyzed) is monitored at two separate time instants denoted as $t$ and $t^{\prime}$. The RIF of the area for both moments is denoted as $\operatorname{RIF}(t)$ and $\operatorname{RIF}\left(t^{\prime}\right)$, respectively. It is assumed that the number of interferers at time instant $t$ is $M$ and at time instant $t^{\prime}$ is $(M+1)$. Without loss of generality, it is additionally assumed that the area of interest is a square with side length $A$. This area, i.e. the RIF, is divided in a mesh of 
smaller and equal square regions, each with a side length $a$. The ratio $A / a$ defines the resolution of the mesh and $\rho=(A / a)^{2}$ denotes the number of regions. The interference level at an arbitrary point $p_{i}$ in the $i$-th region is calculated for both $\operatorname{RIF}(t)$ and $\operatorname{RIF}\left(t^{\prime}\right)$ and denoted as $I_{i}\left(t, p_{i}\right)$ and $I_{i}\left(t^{\prime}, p_{i}\right), i=1, \ldots, \rho$, respectively, and expressed in the $\mathrm{mW}$ scale. The increase of the interference level at an arbitrary point $p_{i}$ in the time interval $\left(t, t^{\prime}\right)$ for each region $i$, due to the appearance of a new interferer in the area of interest, can be obtained by subtracting the interference level at the given point $p_{i}$ in moment $t$ from the interference level at the same point $p_{i}$ in moment $t^{\prime}$ :

$$
\Delta I_{i}\left(p_{i}\right)=I_{i}\left(t^{\prime}, p_{i}\right)-I_{i}\left(t, p_{i}\right), i=1, \ldots, \rho
$$

The average increase of the interference level in the $i$-th region $\Delta I_{i}$ is defined as:

$$
\Delta I_{i}=\frac{1}{L} \sum_{j=1}^{L} \Delta I_{i, j}\left(p_{i, j}\right)
$$

where $L$ denotes the number of points per region. Fig. 3 depicts an example of an active transmitter and its influence on the regions of the RIF. In most of the cases, the average increase of the interference level will be highest in the region that contains the interferer. However, in some cases this can be misleading due to the negative channel effects like shadowing or fading as well as the number and position of the interpolation points. In order to alleviate these negative effects, $\Delta I_{i}$ must be compared to a reference level denoted as Interference Threshold (IT) - $\Delta$. If $\Delta I_{i} \geq \Delta$, then the region $i$ is a possible candidate for interferer holder.

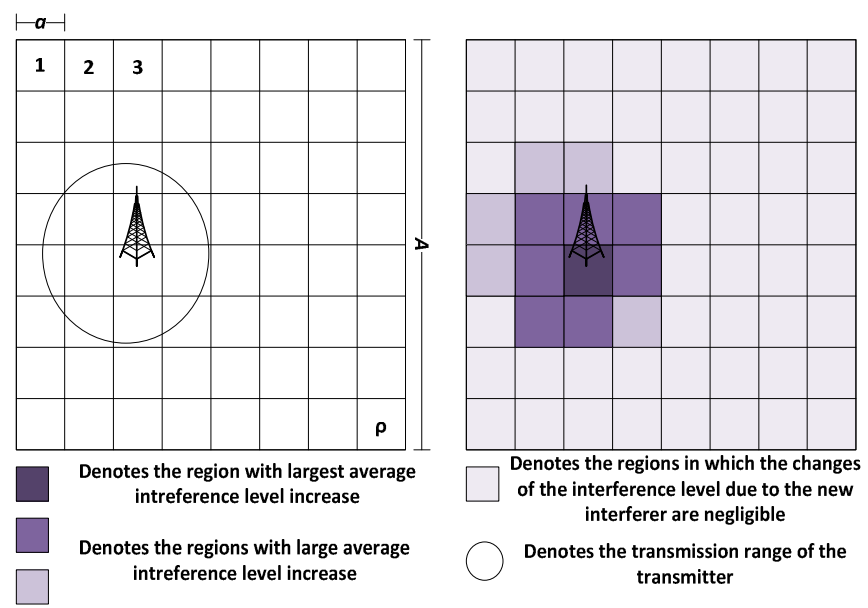

Figure3. Radio transmission range of a single transmitter and influence on the area regions

The value of the IT depends on many aspects such as transmitter power, path loss, number of sensors, interpolation technique fidelity, region size, the location of the interferer within the specific region etc. The proposed localization method considers that $\Delta I_{i}$, i.e. the average increase of the interference level in every region, is a random variable with a PDF denoted as $f_{\Delta I_{i}, r}(x) ; i=1, \ldots, \rho ; r \in k$. The notation assumes that the transmitter is located at an arbitrary point $r$ in the $k$-th region. Thus, the probability of detecting the transmitter in the $k$-th region can then be calculated as:

$$
P\left(\Delta I_{k, r} \geq \Delta \mid k\right)=\int_{\Delta}^{\infty} f_{\Delta I_{k, r}}(x) d x
$$

where $\Delta$ is the IT.

In general, the random variables $\Delta I_{i, r} ; i=1, \ldots, \rho$ are statistically dependent and there exists a certain level of correlation between them. However, in order to provide analytical tractability and simplicity of the mathematical model, this paper assumes that all $\Delta I_{i, r} ; i=1, \ldots, \rho$ are statistically independent random variables. The probability of correct location estimation of the transmitter in the $k$-th region is given with:

$$
P_{D \mid k, r}=P\left(\Delta I_{k, r} \geq \Delta \mid k\right) \prod_{j=1, j \neq k}^{\rho} P\left(\Delta I_{j, r}<\Delta \mid k\right)
$$

where $P\left(\Delta I_{j, r}<\Delta \mid k\right)$ represents the probability of not detecting the interferer in the $j$-th region when the interferer has appeared at an arbitrary point $r$ in the $k$-th region.

The probabilities $P\left(\Delta I_{k, r} \geq \Delta \mid k\right) \quad$ and $P\left(\Delta I_{j, r}<\Delta \mid k\right) ; i, j=1, \ldots, \rho ; j \neq k$ can be calculated in terms of the marginal distributions $f_{\Delta l_{j, r}}(x) ; j=1, \ldots, \rho ; r \in k$ of the random variables $\Delta I_{j, r} ; j=1, \ldots, \rho ; r \in k$. The analytical form of these PDFs is generally unknown, but can be estimated from multiple consecutive measurements, i.e. RIF maps. Fig. 4 shows that the histogram i.e. the ePDF of $\Delta I_{i}$ follows the Normal distribution. Therefore, eq. (4) becomes:

$$
P_{D \mid k, r}=\frac{1}{2} \operatorname{erfc}\left(\frac{\Delta-\mu_{k, r}}{\sigma_{k, r} \sqrt{2}}\right) \prod_{j=1, j \neq k}^{\rho}\left(1-\operatorname{erfc}\left(\frac{\Delta-\mu_{j, r}}{\sigma_{j, r} \sqrt{2}}\right)\right)
$$

where $\mu_{k, r}$ and $\sigma_{k, r}$ denote the mean and variance of the $k$-th region, while $\mu_{j, r}$ and $\sigma_{j, r}$ denote the mean and variance of the remaining regions.

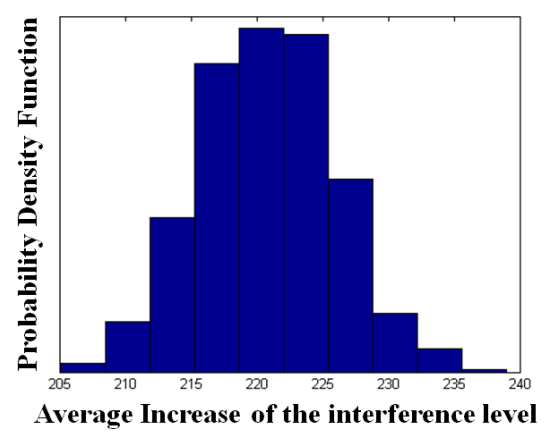

(a) Indoor 


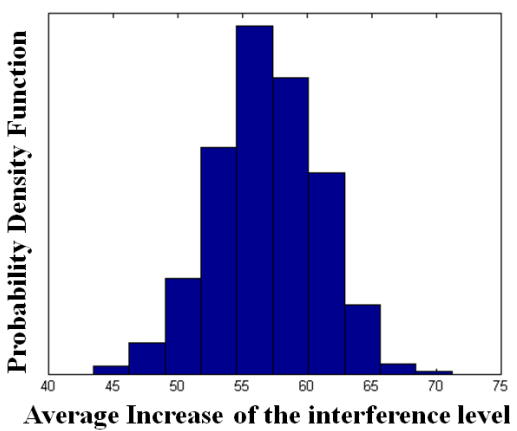

(b) Outdoor

Figure 4. Normalized histogram for the $k$-th region

The probability of correct transmitter localization in the $k$ th region can be calculated by averaging (4) over all possible locations $r$ of the interferer within the given region. Assuming that $r$ is a random variable uniformly distributed over each region (denoting its PDF with $f_{i}(r) ; i=1, \ldots, \rho$ ), the probability of correct interferer detection and localization in the $k$-th region can be calculated as:

$$
P_{D \mid k}=\int_{r e g . k} P_{D \mid k, r} f_{k}(r) d r
$$

where $P_{D \mid i}$ denotes the probability of correct location estimation in region $k$ averaged over all possible interferer locations within the same region. Furthermore, assuming that the interferer can appear in each region $i=1, \ldots, \rho$ with equal probability, the probability of correct interferer detection and localization is given by:

$$
P_{D}=\frac{1}{\rho} \sum_{i=1}^{\rho} P_{D \mid i, j}
$$

The value of $\Delta$ can be calculated from the likelihood ratio of the $\Delta I_{k}$ and $\Delta I_{j}$ PDFs:

$$
\frac{f_{\Delta I_{k, r}}(\Delta)}{f_{\Delta I_{k, j}}(\Delta)}=1
$$

where $k$ denotes the region that contains the transmitter, and the $j$ denotes the region whose PDF has the highest mean i.e. $\max _{j \neq k}\left\{\mu_{j, r}\right\}$. Based on the assumption that the PDFs follow the normal distribution, eq. $5, \Delta$ can be computed as:

$$
e^{-\frac{\left(\Delta-\mu_{k, r}\right)^{2}}{2 \sigma_{k, r}^{2}}} \cdot \sigma_{k, r}^{-1}=e^{-\frac{\left(\Delta-\max _{j \neq k}\left\{\mu_{j, r}\right\}\right)^{2}}{2 \sigma_{j, r}^{2}}} \cdot \sigma_{j, r}^{-1}
$$

\section{B. RIF based localization with movable regions}

The localization approach presented previously is mostly empirically based and it does not require any specific channel knowledge. Hence, the method does not cope with undesired propagation phenomena (e.g. deep fading, hidden terminal problem etc.). Furthermore, the typical scenarios in which the transmitter is located near the edge of the region results in significant increase of the probability of detecting the transmitter in the neighboring regions, thus, according to (4) the performance of the technique deteriorates.

A possible way to mitigate the negative effects of the channel variability and the prediction error introduced by the underlying spatial interpolation technique and to increase the probability of correct location estimation is the usage of nonfixed dynamic area division scheme, referred to as a Moving Interferer Container (MIC) approach. MIC performs quick search for more optimal area division scheme (while keeping the region size fixed) usually by moving and placing the region containing the transmitter maximizing the probability of correct location estimation. The work presented in this paper employs a simple two-step MIC algorithm. Initially, the proposed technique is executed by using fixed area division scheme, which results in identifying the region (denoted with $k$ ) with highest probability of containing the transmitter. Then, the algorithm calculates the probability of location estimation for the neighboring regions and slightly moves region $k$ towards the neighboring region with the highest probability of transmitter detection. This essentially results in a new area division scheme. The algorithm concludes with re-calculating the probability of locating the transmitter for the new area division scheme. As evident in Section V, the performances of the localization technique are drastically improved under the MIC approach.

It is important to note that the introduction of the MIC solution increases the computational complexity of the overall detection and localization technique. However, the performance gain obtained by implementing MIC can justify the increased computational cost especially when operating with low percentage of sensors and large regions. Moreover, the MIC approach allows for design of various different algorithms (e.g. an iterative approach etc.).

\section{PERFORMANCE EVALUATION}

This section gives an insight into the performances of the proposed localization method by analyzing the probability of transmitter location estimation in terms of the number of sensors, channel and error in range estimation. Assuming that the estimated location of the transmitter is positioned in the center of the region, then the maximal error in range estimation will be $\xi=a \sqrt{2} / 2$, where $a$ denotes the side length of the region. To obtain relevant results, Monte Carlo simulations are carried out for all performance metrics. Table 1 lists the used simulation parameters.

TABLE I. SIMULATION PARAMETERS

\begin{tabular}{|c|c|}
\hline \multicolumn{2}{|c|}{ Simulation parameters } \\
\hline Interpolation technique & IDW modified Sheppard's \\
\hline \multicolumn{2}{|c|}{ Indoor parameters } \\
\hline Propagation model & $\begin{array}{c}\text { Multi-wall with log-normal } \\
\text { shadowing }\end{array}$ \\
\hline
\end{tabular}




\begin{tabular}{|c|c|}
\hline \multicolumn{2}{|c|}{ Simulation parameters } \\
\hline Interpolation technique & IDW modified Sheppard's \\
\hline Pathlosss exponent & 3.5 \\
\hline Operating frequency & $2.4 \mathrm{GHz}$ \\
\hline Transmit power & $10 \mathrm{dBm}$ \\
\hline Area side length (A) & $40 \mathrm{~m}$ \\
\hline Initial number of transmitters & 2 \\
\hline \multicolumn{2}{|c|}{ Outdoor parameters } \\
\hline Propagation model & Hata with log-normal \\
shadowing \\
\hline Transmitter height - receiver \\
height
\end{tabular}

Fig. 5 depicts the probability of location estimation $\left(P_{D}\right)$, for different dimensions of the regions, in dependence of the relative number (to the total number of area mesh points) of randomly scattered sensors. The area mesh points represent the unobserved area locations, i.e. the interpolation points. It is evident that the method performs better for larger region dimensions due to the higher error in range estimation. Furthermore, when using the MIC approach, the performance of the method is substantially increased. The results from Fig. 5 pinpoint the possible applicability of the spatial interpolation based location estimation, i.e. scenarios that require only a rough estimation of the location of the new transmitters and the swiftness of the localization is not of the outmost importance (e.g. cognitive femto-cells).

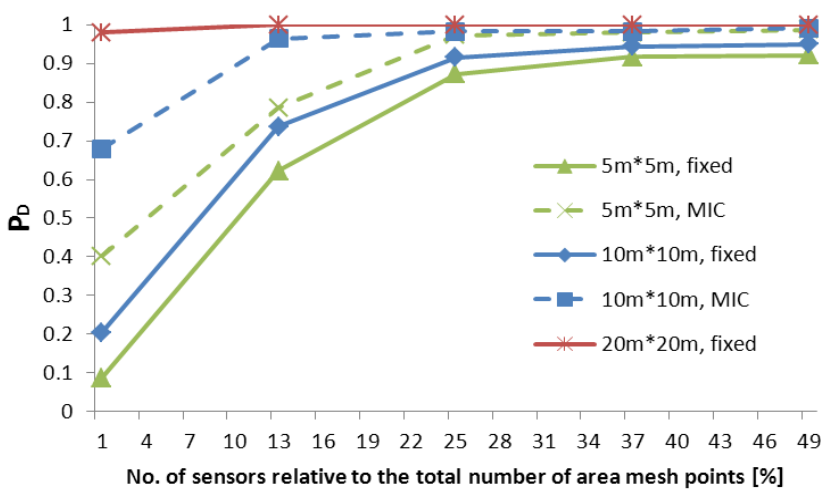

Figure 5. Probability of location estimation vs. number of sensors for indoor environment and scattered positioning (6.5 dB Shadowing Variance)

Fig. 6 depicts the probability of location estimation in dependence of the relative number of sensors (located in a grid). Similar conclusions as for Fig. 5 apply. Additionally, it is evident that the method's performance is not seriously affected by the positioning type (i.e. scattered or gridded) which can be beneficial in many realistic scenarios where the positioning of the sensors is random i.e. scattered.

Fig. 7 gives the dependence of the probability of location estimation on the shadowing variance for indoor environment. Higher level of the shadowing variance significantly decreases the performance of the localization method for smaller regions. It is evident that when the region size is considerably large, the method proves to be resistant to the shadowing effect. Moreover, when utilizing the MIC approach, the method proves to be more resistant for high values of the shadowing effect.

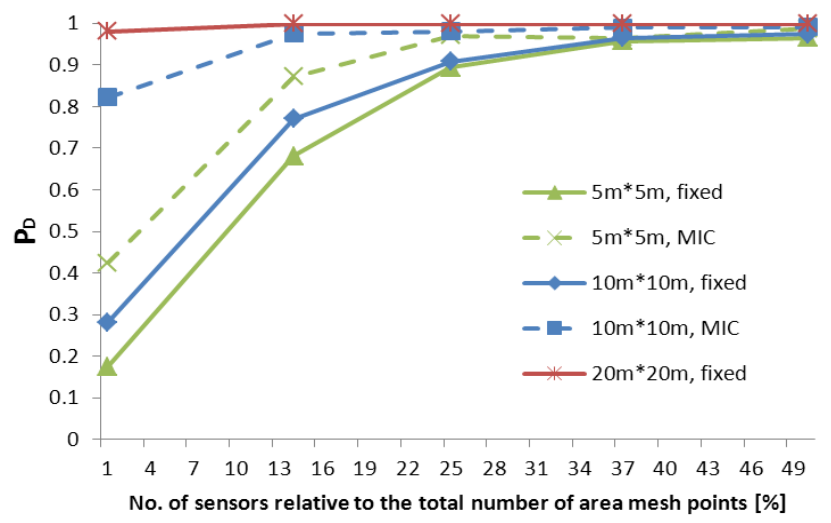

Figure 6. Probability of location estimation vs. number of sensors for indoor environment and gridded positioning of the sensors (6.5 dB Shadowing Variance)

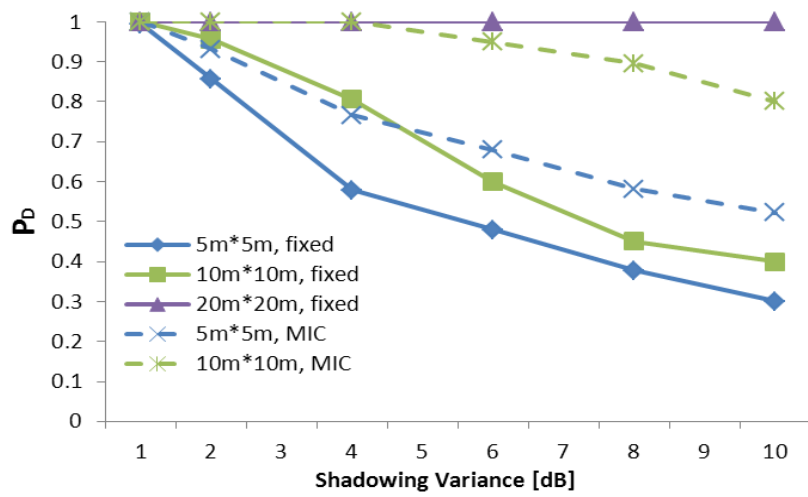

Figure 7. Probability of location estimation vs. shadowing variance for indoor environment and gridded positioning of the sensors $(6.25 \%$ relative number of sensors)

Fig. 8 depicts the probability of location estimation in dependence of the shadowing variance for outdoor environments.

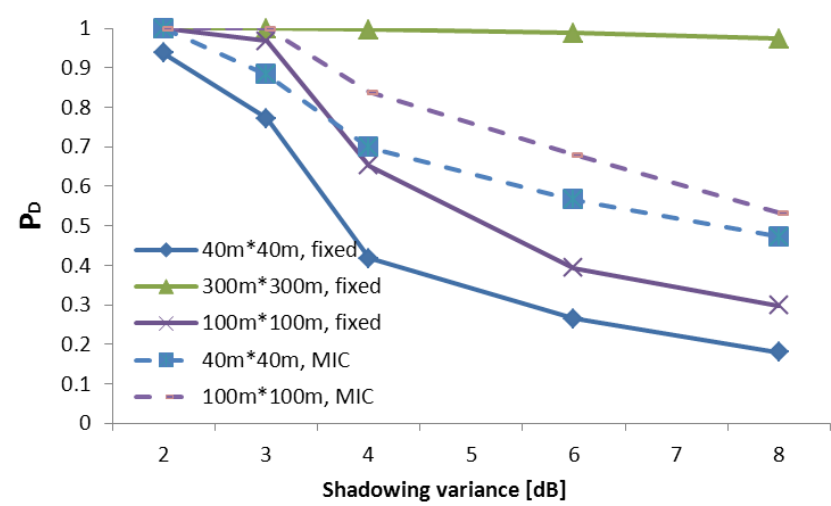

Figure 8. Probability of location estimation vs. shadowing variance for outdoor environment and gridded positioning of the sensors $(6.25 \%$ relative number of sensors) 
It is evident that when using the MIC approach, the proposed method can reliably detect the transmitter in more than $70 \%$ of the cases on a resolution scale of approximately $100 \mathrm{~m}$ for a shadowing variance of $4 \mathrm{~dB}$. This performance is more than adequate when considering an outdoor CR scenario, e.g. opportunistic access on television white spaces (TVWS).

Fig. 9 shows the cumulative distribution function of the error in range estimation for indoor environment. In order to achieve small range estimation error, the method requires high number of sensors.

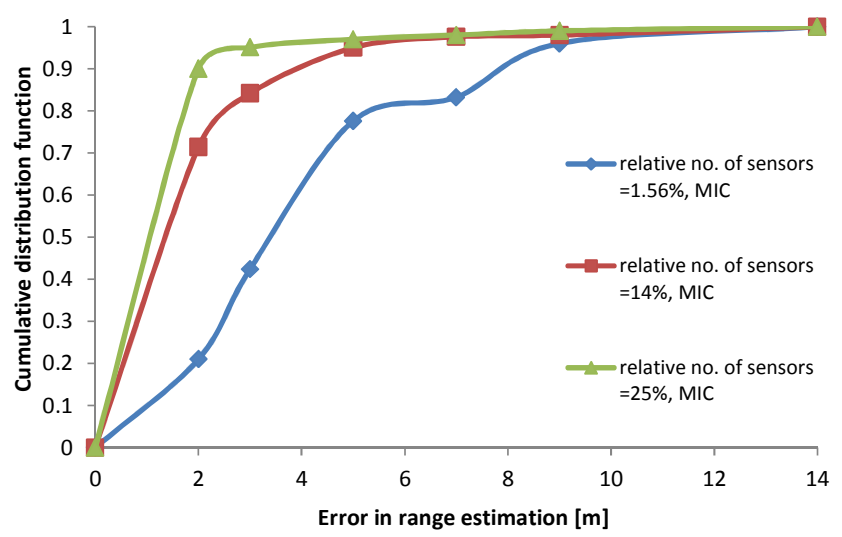

Figure 9. Cumulative distribution function of the error in range estimation for indoor environment ( $6.5 \mathrm{~dB}$ Shadowing Variance)

As evident, the proposed method can reliably detect the transmitter in more than $70 \%$ of the cases on a resolution scale of approximately $5 \mathrm{~m}$ (a typical room) for a relative number of sensors below $2 \%$. In terms of a femto-cell scenario, this can be interpreted as the capability of the femto-cells to detect a new transmitter. For example, if every apartment in a building has one femto-cell capable of RSS measurements and a new transmitter becomes active, then the proposed method will detect the transmitter on a scale of a room in more than $70 \%$ of the time and on a scale of an apartment (resolution of more than $9 \mathrm{~m}$ ) in more than $99 \%$ of the time.

\section{CONCLUSION}

Detection of unused spectrum allows CR users to opportunistically reuse the available spectrum and alleviate the spectrum scarcity problem. The process of transmitter localization can increase the radio environmental awareness of the CR systems and improve the overall spectrum efficiency.

This paper proposes a novel method for location estimation of radio transmitters in a $\mathrm{CR}$ based environment. It utilizes the RIF maps obtained by interpolating RSS measurement data from a number of sparsely distributed sensors. Unlike most of the existing localization algorithms, the proposed method is computationally efficient and does not depend on complex hardware solutions (e.g. antenna arrays, high fidelity synchronization etc.). The computational efficiency comes in trade-off with the localization precision of the method, however the results show that its performance is suitable for CR scenarios (e.g. cognitive femto-cells, TVWS etc.). Main limitation of the method is the fidelity of the underlying interpolation technique and the introduced interpolation error.

Future work will focus on detailed assessment of different interpolation techniques and their impact on the precision of the method. It will also investigate the effect of variety of scenarios targeting different propagation models as well as the number and distribution of the transmitters.

\section{ACKNOWLEDGMENT}

This work was funded by the EC project QUASAR (FP7248303) [10] and inspired by the EC project ACROPOLIS (FP7-257626) [11]. The authors would like to thank everyone involved. The authors also extend their gratitude to the COST IC0902 action [12].

\section{REFERENCES}

[1] D J. K. Nelson, M. Hazen, and M. R. Gupta, "Global optimization for multiple transmitter localization,” In Proc. of MILCOM, Oct. 2006.

[2] S. Haykin, K. J. R. Liu, Handbook on Array Processing and Sensor Networks. John Wiley and Sons, Inc, 2009.

[3] T. He, J. A. Stankovic, C. Huang, T. Abdelzaher, and B. M. Blum, "Range-Free Localization Schemes for Large Scale Sensor Networks," In Proc. of MOBICOM, pp 51-62, 2007.

[4] Ouyang, R.W., Wong, A.K.-S., Chin-Tau Lea, "Received Signal Strength-Based Wireless Localization via Semidefinite Programming: Noncooperative and Cooperative Schemes," IEEE Transactions on wireless communications, vol. 59, no. 3, Jan. 2010.

[5] G. Wang, and K. Yang, "A New Approach to Sensor Node Localization Using RSS Measurements in Wireless Sensor Networks," IEEE Transactions on wireless communications, vol. 10, no. 5, May. 2011

[6] Q. Zhang, C. H. Foh, B. Seet, and A. C. M. Fong, "RSS Ranging Based Wi-Fi Localization for Unknown Path Loss Exponent" in Proc. of GLOBECOM, pp 1-5, 2011.

[7] L. Lin, and H. C. So, "Best Linear Unbiased Estimator Algorthm for Received Signal Strength Based Localization", in Proc. of EUSIPCO 2011.

[8] R. K. Martin, R. Thomas, "Algorithms and Bounds for Estimating Location, Directionality, and Environmental Parameters of Primary Spectrum Users," IEEE Transactions on wireless communications, vol. 8, no. 11, Nov. 2009.

[9] R. J. Renka, "Multivariate Interpolation of Large Sets of Scattered Data," ACM Transactions on Mathematical Software, vol. 14, no. 2, pp 139-148, June 1988.

[10] EC FP7 project QUASAR, Information available at: http://www.quasarspectrum.eu/.

[11] EC FP7 project ACROPOLIS, Information available at: http://www.ictacropolis.eu/.

[12] COST Action IC0902: "Cognitive Radio and Networking for Cooperative Coexistence of Heterogeneous Wireless Networks". Information available at: http://newyork.ing.uniroma1.it/IC0902. 